PROCEEDINGS OF THE

AMERICAN MATHEMATICAL SOCIETY

Volume 130, Number 6, Pages 1673-1679

S 0002-9939(01)06206-2

Article electronically published on October 5, 2001

\title{
ASYMPTOTICS FOR THE HEAT EQUATION IN THE EXTERIOR OF A SHRINKING COMPACT SET IN THE PLANE VIA BROWNIAN HITTING TIMES
}

\author{
ROSS G. PINSKY
}

(Communicated by Claudia M. Neuhauser)

\begin{abstract}
Let $D_{r}=\left\{x \in R^{2}:|x| \leq r\right\}$ and let $\gamma$ be a continuous, nonincreasing function on $[0, \infty)$ satisfying $\lim _{t \rightarrow \infty} \gamma(t)=0$. Consider the heat equation in the exterior of a time-dependent shrinking disk in the plane:

$$
\begin{aligned}
& u_{t}=\frac{1}{2} \Delta u, x \in R^{2}-D_{\gamma(t)}, t>0, \\
& u(x, 0)=0, x \in R^{2}-D_{\gamma(t)}, \\
& u(x, t)=1, x \in D_{\gamma(t)}, t>0 .
\end{aligned}
$$

If there exist constants $0<c_{1}<c_{2}$ and a constant $k>0$ such that $c_{1} t^{-k} \leq$ $\gamma(t) \leq c_{2} t^{-k}$, for sufficiently large $t$, then $\lim _{t \rightarrow \infty} u(x, t)=\frac{1}{1+2 k}$. The same result is also shown to hold when $D_{\gamma(t)}$ is replaced by $L_{\gamma(t)}$, where $L_{r}=$ $\left\{\left(x_{1}, 0\right) \in R^{2}:\left|x_{1}\right| \leq r\right\}$. Also, a discrepancy is noted between the asymptotics for the above forward heat equation and the corresponding backward one. The method used is probabilistic.
\end{abstract}

\section{Statement of Results}

Let

$$
D_{r}=\left\{x \in R^{d}:|x| \leq r\right\}
$$

and consider the heat equation in the exterior domain $R^{d}-D_{1}$ :

$$
\begin{aligned}
& w_{t}=\frac{1}{2} \Delta w,(x, t) \in\left(R^{d}-D_{1}\right) \times(0, \infty), \\
& w(x, 0)=0, x \in R^{d}-D_{1}, \\
& w(x, t)=1,(x, t) \in D_{1} \times[0, \infty) .
\end{aligned}
$$

The asymptotic behavior of the solution $w(x, t)$ for $x \in R^{d}-D_{1}$ as $t \rightarrow \infty$ is well known:

$$
\lim _{t \rightarrow \infty} w(x, t)= \begin{cases}|x|^{2-d}, & \text { if } d \geq 3 \\ 1, & \text { if } d=1,2\end{cases}
$$

Received by the editors May 20, 2000 and, in revised form, November 22, 2000.

1991 Mathematics Subject Classification. Primary 35K05, 35B40, 60J65.

Key words and phrases. Heat equation, planar Brownian motion, hitting times, modulus of Brownian motion, large time asymptotics.

This research was supported by the Fund for the Promotion of Research at the Technion. 
In particular, this shows that a heat source in the shape of a ball does not fully heat three-dimensional space, whereas a heat source in the shape of a disk does fully heat the plane.

The question we address here is this: how effective will the heating be in the plane if the heat source shrinks in time? That is, instead of having a temporally constant heat source $D_{1}$, we use a heat source $D_{\gamma(t)}$, where $\gamma$ is a continuous, positive function decreasing to 0 as $t \rightarrow \infty$. We have the following equation:

$$
\begin{aligned}
& u_{t}=\frac{1}{2} \Delta u, x \in R^{2}-D_{\gamma(t)}, t>0, \\
& u(x, 0)=0, x \in R^{2}-D_{\gamma(t)}, \\
& u(x, t)=1, x \in D_{\gamma(t)}, t \geq 0 .
\end{aligned}
$$

If $\lim _{t \rightarrow \infty} u(x, t)$ exists, then it should be harmonic in $R^{2}-\{0\}$, and since the only bounded harmonic functions in this domain are the constants, it follows that the above limit, if it exists, should be equal to a constant $c \in[0,1]$. Incidentally, the same type of argument shows that the corresponding problem in higher dimensions is uninteresting: if the limit exists, it should be harmonic in $R^{d}-0$, and dominated by $|x|^{2-d}$; the only such bounded, nonnegative function is 0 . A completely rigorous argument can easily be made using the maximum principle.

Before stating the theorem, we derive a probabilistic representation for the solution $u$ to (1.2). We recall a fundamental property of Brownian motion: let $P_{x}$ denote Wiener measure for a standard Brownian motion $B(t)$ in $R^{d}$ starting from $x \in R^{d}$ and let $E_{x}$ denote the corresponding expectation. Let $G \subset R^{d}$ be a domain and let $\sigma_{G^{c}}=\inf \left\{t \geq 0: B(t) \in G^{c}\right\}$ denote the first entrance time into $G^{c}$. If $W(x, s)$ is twice continuously differentiable for $x \in G$, once continuously differentiable for $s \in(0, t]$, and continuous on $\bar{G} \times(0, t] \cup G \times[0, t]$, and if $\frac{1}{2} \Delta W+\frac{\partial W}{\partial s}$ is bounded on $G \times(0, t]$, then $W\left(B\left(s \wedge \sigma_{G^{c}}\right), s \wedge \sigma_{G^{c}}\right)-\int_{0}^{s \wedge \sigma_{G^{c}}}\left(\frac{1}{2} \Delta W+\frac{\partial W}{\partial s^{\prime}}\right)\left(B\left(s^{\prime}\right), s^{\prime}\right) d s^{\prime}$ is a martingale under $P_{x}$ for $s \in[0, t]$. In particular, if it so happens that $\frac{1}{2} \Delta W+\frac{\partial W}{\partial s} \equiv 0$ in $G \times(0, t)$, then $W\left(B\left(s \wedge \sigma_{G^{c}}\right), s \wedge \sigma_{G^{c}}\right)$ is a martingale. Since the expectation of a martingale is constant in time, equating the value of the expectation when $s=0$ with the value for $s=t$, we obtain the formula

$$
W(x, 0)=E_{x} W\left(B\left(t \wedge \sigma_{G^{c}}\right), t \wedge \sigma_{G^{c}}\right), \text { for } x \in G .
$$

Equation (1.3) leads to the well-known probabilistic representation for $w(x, t)$. Fix $t>0$ and apply (1.3) to $W(x, s)=w(x, t-s)$ and $G=R^{d}-D_{1}$. From the boundary condition and the initial condition in (1.1) it follows that

$$
W\left(B\left(t \wedge \sigma_{G^{c}}\right), t \wedge \sigma_{G^{c}}\right)=w\left(B\left(t \wedge \sigma_{D_{1}}\right), t-t \wedge \sigma_{D_{1}}\right)=1
$$

on the event $\left\{\sigma_{D_{1}}<t\right\}$, while

$$
W\left(B\left(t \wedge \sigma_{G^{c}}\right), t \wedge \sigma_{G^{c}}\right)=w\left(B\left(t \wedge \sigma_{D_{1}}\right), t-t \wedge \sigma_{D_{1}}\right)=0
$$

on the event $\left\{\sigma_{D_{1}}>t\right\}$. Since $P_{x}\left(\sigma_{D_{1}}=t\right)=0$, we obtain from (1.3) that

$$
w(x, t)=P_{x}\left(\sigma_{D_{1}} \leq t\right) \text { for } x \in R^{d}-D_{1} .
$$

From (1.4), it follows that the dichotomy between $\lim _{t \rightarrow \infty} w(x, t)=1$ for $d=1,2$ and $\lim _{t \rightarrow \infty} w(x, t)<1$ for $d \geq 3$ which we observed above is just the dichotomy between recurrence and transience of Brownian motion. 
In order to apply (1.3) to the solution $u$ of (1.2), we define for each $t>0$ the stopping time

$$
\sigma_{\gamma}^{(t)}=\inf \left\{s \in[0, t]: B(s) \in D_{\gamma(t-s)}\right\}
$$

with the convention that $\sigma_{\gamma}^{(t)}=\infty$ if $\left\{s \in[0, t]: B(s) \in D_{\gamma(t-s)}\right\}$ is empty. Fixing a $t>0$, consider the function $W(x, s) \equiv u(x, t-s), \quad 0 \leq s \leq t, x \in$ $R^{2}-D_{\gamma(t-s)}$. By (1.2), we have $\left(\frac{1}{2} \Delta W+\frac{\partial W}{\partial s}\right)(x, s) \equiv 0$ for $x \in R^{2}-D_{\gamma(t-s)}$ and $0 \leq s \leq t$. We now apply (1.4) to this choice of $W(x, s)$ along with the stopping time $\sigma_{\gamma}^{(t)}$. The fact that the domain is time-dependent does not cause any problem. Note from the boundary condition and the initial condition in (1.2) that $W\left(B\left(t \wedge \sigma_{\gamma}^{(t)}\right), t \wedge \sigma_{\gamma}^{(t)}\right)=u\left(B\left(t \wedge \sigma_{\gamma}^{(t)}\right), t-t \wedge \sigma_{\gamma}^{(t)}\right)=1$ on the event $\left\{\sigma_{\gamma}^{(t)}<t\right\}$, while $W\left(B\left(t \wedge \sigma_{\gamma}^{(t)}\right), t \wedge \sigma_{\gamma}^{(t)}\right)=u\left(B\left(t \wedge \sigma_{\gamma}^{(t)}\right), t-t \wedge \sigma_{\gamma}^{(t)}\right)=0$ on the event $\left\{\sigma_{\gamma}^{(t)}>t\right\}$. Since $P_{x}\left(\sigma_{\gamma}^{(t)}=t\right)=0$, we obtain the following probabilistic representation for the solution $u$ of $(1.2)$ :

$$
u(x, t)=P_{x}\left(\sigma_{\gamma}^{(t)} \leq t\right)=P_{x}\left(B(s) \in D_{\gamma(t-s)} \text { for some } s \in[0, t]\right) .
$$

We will prove the following theorem.

Theorem. Let $u(x, t)$ be the solution to (1.2), where $\gamma(t)$ is a continuous, nonincreasing function. Assume that there exist constants $0<c_{1}<c_{2}$ and a constant $k>0$ such that $c_{1} t^{-k} \leq \gamma(t) \leq c_{2} t^{-k}$ for sufficiently large $t$. Then

$$
\lim _{t \rightarrow \infty} u(x, t)=\frac{1}{1+2 k} .
$$

Remark. By a basic monotonicity property which is an obvious consequence of the maximum principle, it follows that if $\gamma(t)$ decreases to 0 faster than any negative power of $t$, then $\lim _{t \rightarrow \infty} u(x, t)=0$, while if $\gamma(t)$ decreases to 0 more slowly than any negative power of $t$, then $\lim _{t \rightarrow \infty} u(x, t)=1$.

With just a little extra work, we will prove the same result when $D_{r}$ is replaced by a one-dimensional set. Let

$$
L_{r}=\left\{\left(x_{1}, 0\right) \in R^{2}:\left|x_{1}\right| \leq r\right\} .
$$

Corollary. Let $U(x, t)$ denote the solution to (1.2) with $D_{\gamma(t)}$ replaced by $L_{\gamma(t)}$. Then (1.6) holds with $u$ replaced by $U$.

Remark. By the basic monotonicity property, the same result also holds if $D_{\gamma(t)}$ or $L_{\gamma(t)}$ is replaced by $C_{\gamma(t)}$ where $r \rightarrow C_{r}$ is a continuous map from $[0, \infty)$ to the compact subsets of $R^{2}$, satisfying $L_{r} \subset C_{r} \subset D_{r}$.

There turns out to be an interesting discrepancy between the asymptotic behavior of the solution $u$ to the forward heat equation (1.2) and the solution to the corresponding backward heat equation. Let $v^{(t)}(x, s)$ denote the solution to the backward heat equation

$$
\begin{aligned}
& v_{s}^{(t)}+\frac{1}{2} \Delta v^{(t)}=0, x \in R^{2}-D_{\gamma(s)}, 0 \leq s \leq t, \\
& v^{(t)}(x, t)=0, x \in R^{2}-D_{\gamma(t)}, \\
& v^{(t)}(x, s)=1, x \in D_{\gamma(s)}, 0 \leq s \leq t .
\end{aligned}
$$


Consider the stopping time

$$
\sigma_{\gamma}=\inf \left\{t \geq 0: B(t) \in D_{\gamma(t)}\right\}
$$

and define

$$
v(x, t)=P_{x}\left(\sigma_{\gamma} \leq t\right)
$$

An analysis similar to that used to obtain the probabilistic representation of $u$ in (1.5) shows that $v(x, t)=v^{(t)}(x, 0)$. (Use (1.3) with $W(x, s)=v_{s}^{t}(x, s), 0 \leq s \leq t$, and use $\sigma_{\gamma}$ in place of $\sigma_{G^{c}}$.) An old result of Spitzer [3] indicates that for $|x|>\gamma(0)$,

$$
P_{x}\left(\frac{|B(t)|}{\gamma(t)} \leq 1 \text { for some } t \geq 0\right)=1 \text { if and only if } \int^{\infty} \frac{1}{t|\log \gamma(t)|} d t=\infty .
$$

In terms of $v^{(t)},(1.7)$ states that

$$
\begin{aligned}
& \lim _{t \rightarrow \infty} v^{(t)}(x, 0)=\lim _{t \rightarrow \infty} P_{x}\left(B(s) \in D_{\gamma(s)} \text { for some } s \in[0, t]\right)=1 \\
& \text { if and only if } \int^{\infty} \frac{1}{t|\log \gamma(t)|} d t=\infty
\end{aligned}
$$

Note, for example, that the integral in (1.8) will be infinite if $\gamma(t) \geq t^{-\log \log t}$, but not if $\gamma(t) \leq t^{-(\log \log t)^{1+\epsilon}}$. In contrast, by Theorem 1, it follows that

$$
\begin{aligned}
& \lim _{t \rightarrow \infty} u(x, t) \\
& =\lim _{t \rightarrow \infty} P_{x}\left(B(s) \in D_{\gamma(t-s)}, \text { for some } s \in[0, t]\right) \begin{cases}=1, & \text { if } \lim _{t \rightarrow \infty} t^{k} \gamma(t)=\infty, \\
& \text { for all } k>0, \\
& \text { if } \lim _{t \rightarrow \infty} t^{k} \gamma(t)=0, \\
& \text { for some } k>0 . \\
=0, & \text { if } \lim _{t \rightarrow \infty} t^{k} \gamma(t)=0, \\
\text { for all } k>0 .\end{cases}
\end{aligned}
$$

Thus, there is a discrepancy between the asymptotic behavior of the solutions to the forward and backward heat equations. In probabilistic terms, there is a discrepancy in the asymptotic behavior of the hitting times of a shrinking disk, depending on whether the shrinking occurs in forward time or backward time. In particular, note that if $\gamma(t)$ decays to 0 faster than any negative power of $t$, but $\int^{\infty} \frac{1}{t|\log \gamma(t)|} d t=\infty$, as occurs for example if $\gamma(t)=t^{-\log \log t}$, then (1.8) is equal to 1 while (1.9) is equal to 0 .

\section{Proof of the Theorem and Corollary}

Since everything concerning the Theorem is radially symmetric, we will use the notation $P_{r}, r \geq 0$, instead of $P_{x}, x \in R^{2}$, where $r=|x|$. When we turn to the proof of the Corollary, we will return to the notation $P_{x}$. We will need the following Lemma. 
Lemma. Let $\tau_{a}=\inf \{t \geq 0:|X(t)|=a\}$. For $l_{1}, l_{2}$ satisfying $0<l_{1}<\frac{1}{2}<l_{2}$, and for $0<a \leq b \leq \frac{1}{2} t^{l_{2}}$, there exists a universal constant $\lambda>0$ such that

$$
\begin{aligned}
& \frac{\log b-\log a}{l_{2} \log t-\log a}-\frac{1}{\lambda} \exp \left(-\lambda t^{2 l_{2}-1}\right) \\
& \quad \leq P_{b}\left(\tau_{a}>t\right) \leq \frac{\log b-\log a}{l_{1} \log t-\log a}+\frac{1}{\lambda} \exp \left(-\lambda t^{1-2 l_{1}}\right) .
\end{aligned}
$$

Proof. Since $P_{b}\left(\tau_{a}>\tau_{c}\right)=\frac{\log b-\log a}{\log c-\log a}$, for $a<b<c$ [2, p. 38], we have for $t, l>0$ such that $b<t^{l}$,

$$
P_{b}\left(\tau_{a}>t\right) \leq P_{b}\left(\tau_{a}>\tau_{t^{l}}\right)+P_{b}\left(\tau_{t^{l}}>t\right)=\frac{\log b-\log a}{l \log t-\log a}+P_{b}\left(\tau_{t^{l}}>t\right)
$$

and

$$
P_{b}\left(\tau_{a}>t\right) \geq P_{b}\left(\tau_{a}>\tau_{t^{l}}\right)-P_{b}\left(\tau_{t^{l}}<t\right)=\frac{\log b-\log a}{l \log t-\log a}-P_{b}\left(\tau_{t^{l}}<t\right) .
$$

The Brownian scaling property gives

$$
P_{b}\left(\tau_{t^{l}}>t\right)=P_{b t^{-l}}\left(\tau_{1}>t^{1-2 l}\right) \text { and } P_{b}\left(\tau_{t^{l}}<t\right)=P_{b t^{-l}}\left(\tau_{1}<t^{1-2 l}\right) .
$$

If $l \in\left(0, \frac{1}{2}\right)$, then $P_{b t^{-l}}\left(\tau_{1}>t^{1-2 l}\right) \leq \frac{1}{\lambda} \exp \left(-\lambda t^{1-2 l}\right)$ for some $\lambda>0$ [1, Theorem 3.6.1], while if $l>\frac{1}{2}$ and $b \leq \frac{1}{2} t^{l}$, then $P_{b t^{-l}}\left(\tau_{1}<t^{1-2 l}\right) \leq \frac{1}{\lambda} \exp \left(-\lambda t^{2 l-1}\right)$ for some $\lambda>0$ [1] Theorem 2.2.2]. The Lemma follows from these estimates.

Proof of the Theorem. By the basic monotonicity property alluded to in the Remark following the Theorem, we can assume without loss of generality that $\gamma(t)=$ $c(1+t)^{-k}$ for some $c>0$ and $k>0$. In light of (1.5), to prove the Theorem we must show that

$$
\lim _{t \rightarrow \infty} P_{b}\left(|B(s)| \leq c(1+t-s)^{-k} \text { for some } s \in[0, t]\right)=\frac{1}{1+2 k} .
$$

Using the right-hand inequality in the Lemma and the monotonicity of $c(1+t)^{-k}$, we have for large $t$,

$$
\begin{aligned}
& P_{b}\left(|B(s)| \leq c(1+t-s)^{-k} \text { for some } s \in[0, t]\right) \geq P_{b}\left(\tau_{c(1+t)^{-k}} \leq t\right) \\
& =1-P_{b}\left(\tau_{c(1+t)^{-k}}>t\right) \geq 1-\frac{\log b-\log c+k \log (1+t)}{l_{1} \log t-\log c+k \log (1+t)}-\frac{1}{\lambda} \exp \left(-\lambda t^{1-2 l_{1}}\right) .
\end{aligned}
$$

Letting $t \rightarrow \infty$ in (2.2) and then letting $l_{1}$ increase to $\frac{1}{2}$, we obtain

$$
\liminf _{t \rightarrow \infty} P_{b}\left(|B(s)| \leq c(1+t-s)^{-k} \text { for some } s \in[0, t]\right) \geq \frac{1}{1+2 k} .
$$

Applying the Markov property at time $\frac{t}{2}$, and using the monotonicity of $c(1+t)^{-k}$ again, we have

$$
P_{b}\left(|B(s)|>c(1+t-s)^{-k} \text { for all } s \in[0, t]\right) \geq E_{b}\left(P_{\left|B\left(\frac{t}{2}\right)\right|}\left(\tau_{c}>\frac{t}{2}\right) ; \tau_{c\left(1+\frac{t}{2}\right)^{-k}}>\frac{t}{2}\right) .
$$

A direct calculation shows that there exists a constant $C>0$, depending on $b$, such that for any $\epsilon>0$,

$$
P_{b}\left(\left|B\left(\frac{t}{2}\right)\right| \leq \epsilon t^{\frac{1}{2}}\right) \leq C \epsilon^{2} \text { for all } t>0 .
$$


Thus, since $P_{b}\left(\tau_{c}>\frac{t}{2}\right)$ is increasing in $b$ for $b>c$, we obtain from (2.4) and (2.5) that for any $\epsilon>0$ and sufficiently large $t$

$$
\begin{aligned}
& P_{b}\left(|B(s)|>c(1+t-s)^{-k} \text { for all } s \in[0, t]\right) \\
& \quad \geq P_{b}\left(\tau_{c\left(1+\frac{t}{2}\right)^{-k}}>\frac{t}{2}\right) P_{\epsilon t^{\frac{1}{2}}}\left(\tau_{c}>\frac{t}{2}\right)-C \epsilon^{2} .
\end{aligned}
$$

Using the lower bound in the Lemma to estimate the two probabilities on the right-hand side of (2.6), we have for sufficiently large $t$,

$$
\begin{aligned}
& \left.P_{b}\left(|B(s)|>c(1+t-s)^{-k}\right) \text { for all } s \in[0, t]\right) \\
& \geq\left(\frac{\log b-\log c+k \log \left(1+\frac{t}{2}\right)}{l_{2} \log \frac{t}{2}-\log c+k \log \left(1+\frac{t}{2}\right)}-\frac{1}{\lambda} \exp \left(-\lambda t^{2 l_{2}-1}\right)\right) \\
& \times\left(\frac{\log \epsilon+\frac{1}{2} \log t-\log c}{l_{2} \log \frac{t}{2}-\log c}-\frac{1}{\lambda} \exp \left(-\lambda t^{2 l_{2}-1}\right)\right)-C \epsilon^{2} .
\end{aligned}
$$

Letting $t \rightarrow \infty$ in (2.7), then letting $\epsilon$ decrease to 0 and $l_{2}$ decrease to $\frac{1}{2}$ gives

$$
\liminf _{t \rightarrow \infty} P_{b}\left(|B(s)|>c(1+t-s)^{-k} \text { for all } s \in[0, t]\right) \geq \frac{2 k}{1+2 k} .
$$

Now (2.1) follows from (2.3) and (2.8).

Proof of the Corollary. We now return to the notation $P_{x}, x \in R^{2}$. Let $\tau_{L_{a}}=$ $\inf \left\{t \geq 0: B(t) \in L_{a}\right\}$. We will show that there exists a constant $K>0$ such that for $0<a<|x|<c$,

$$
\frac{\log |x|-\log a}{\log c-\log a} \leq P_{x}\left(\tau_{L_{a}}>\tau_{c}\right) \leq \frac{\log |x|-\log \frac{a}{2}+K}{\log c-\log \frac{a}{2}}
$$

Using (2.9), it follows immediately from the proof of the Lemma that

$$
\begin{aligned}
& \frac{\log |x|-\log a}{l_{2} \log t-\log a}-\frac{1}{\lambda} \exp \left(-\lambda t^{2 l_{2}-1}\right) \\
& \quad \leq P_{x}\left(\tau_{L_{a}}>t\right) \leq \frac{\log |x|-\log \frac{a}{2}+K}{l_{1} \log t-\log \frac{a}{2}}+\frac{1}{\lambda} \exp \left(-\lambda t^{1-2 l_{1}}\right) .
\end{aligned}
$$

One now proves the Corollary just as the Theorem was proved, using the estimate (2.10) in place of the estimate in the Lemma.

It remains to prove (2.9). The left-hand inequality in (2.9) of course follows trivially since $P_{x}\left(\tau_{L_{a}}>\tau_{c}\right) \geq P_{x}\left(\tau_{a}>\tau_{c}\right)=\frac{\log |x|-\log a}{\log c-\log a}$. For the right-hand inequality, we begin by using the strong Markov property to write

$$
P_{x}\left(\tau_{L_{a}}<\tau_{c}\right) \geq E_{x}\left(P_{B\left(\tau_{\frac{a}{2}}\right)}\left(\tau_{L_{a}}<\tau_{c}\right) ; \tau_{\frac{a}{2}}<\tau_{c}\right) .
$$

For any $t>0$ and $y \in R^{2}$ with $|y|=\frac{1}{2}$, we have $P_{y}\left(\tau_{L_{1}}<t \wedge \tau_{1}\right)>0$. This can be proved in any number of ways; for instance, by applying the Stroock-Varadhan support theorem [1, Theorem 2.6.1], or by applying the reflection principle [2] to the second coordinate of the Brownian motion and using the independence of the two components. Since Brownian motion is a Feller process, it follows that $P_{y}\left(\tau_{L_{1}}<t \wedge \tau_{1}\right)$ is continuous in $y$ [1, Theorem 1.3.1]; thus,

$$
\inf _{y \in R^{2}:|y|=\frac{1}{2}} P_{y}\left(\tau_{L_{1}}<t \wedge \tau_{1}\right)>0
$$


and a fortiori there exists a $\rho>0$ such that $\inf _{y \in R^{2}:|y|=\frac{1}{2}} P_{y}\left(\tau_{L_{1}}<\tau_{1}\right) \geq \rho$. By Brownian scaling it then follows that

$$
P_{y}\left(\tau_{L_{a}}<\tau_{a}\right) \geq \rho>0 \text { for }|y|=\frac{a}{2} \text { and all } a>0 .
$$

Also,

$$
P_{y}\left(\tau_{\frac{a}{2}}<\tau_{c}\right)=\frac{\log a-\log c}{\log \frac{a}{2}-\log c} \equiv q_{a, c} \text { for }|y|=a .
$$

We use (2.12), (2.13) and the strong Markov property to estimate $P_{y}\left(\tau_{L_{a}}<\tau_{c}\right)$ for $|y|=\frac{a}{2}$. Consider the event $\tau_{L_{a}}>\tau_{c}$ under $P_{y}$ with $|y|=\frac{a}{2}$. In order for this event to occur, first of all, starting from $y \in \partial D_{\frac{a}{2}}, B(t)$ must hit $\partial D_{a}$ before hitting $L_{a}$, and this event occurs with probability no greater than $1-\rho$. Then, starting from $\partial D_{a}$, the Brownian motion has a probability $q_{a, c}$ of returning to $\partial D_{\frac{a}{2}}$ before reaching $\partial D_{c}$ (during which time it may hit $L_{a}$, but we ignore this), in which case it gets another chance, starting from $\partial D_{\frac{a}{2}}$, to hit $L_{a}$ before hitting $\partial D_{a}$, etc. This reasoning gives the estimate

$$
P_{y}\left(\tau_{L_{a}}>\tau_{c}\right) \leq \sum_{n=0}^{\infty}(1-\rho)^{n+1} q_{a, c}^{n}\left(1-q_{a, c}\right)=\frac{\left(1-q_{a, c}\right)(1-\rho)}{1-(1-\rho) q_{a, c}} \equiv Q_{a, c} \text { for }|y|=\frac{a}{2} .
$$

From $(2.11),(2.14)$, and the fact that $P_{x}\left(\tau_{\frac{a}{2}}<\tau_{c}\right)=\frac{\log |x|-\log c}{\log \frac{a}{2}-\log c}$, we have

$$
\begin{aligned}
& P_{x}\left(\tau_{L_{a}}>\tau_{c}\right) \leq 1-E_{x}\left(P_{B\left(\tau_{\frac{a}{2}}\right)}\left(\tau_{L_{a}}<\tau_{c}\right) ; \tau_{\frac{a}{2}}<\tau_{c}\right) \leq 1-\left(1-Q_{a, c}\right) \frac{\log |x|-\log c}{\log \frac{a}{2}-\log c} \\
& =\frac{\log |x|-\log \frac{a}{2}+Q_{a, c}(\log c-\log |x|)}{\log c-\log \frac{a}{2}} .
\end{aligned}
$$

From (2.13) and (2.14), we have

$$
Q_{a, c}=\frac{(1-\rho) \log 2}{\log 2+\rho \log \frac{c}{a}} .
$$

It follows from (2.16) that there exists a constant $K>0$ such that $Q_{a, c} \log c \leq K$. Substituting this estimate in the right-hand side of (2.15) proves (2.9).

\section{REFERENCES}

1. Pinsky, R.G., Positive Harmonic Functions and Diffusion, Cambridge Univ. Press, 1995. MR 96m:60179

2. Rogers, L.C.G. and Williams, D., Diffusions, Markov Processes and Martingales, Vol. 1, 2nd ed., Cambridge Univ. Press, 2000. CMP 2001:04

3. Spitzer, F., Some theorems concerning two-dimensional Brownian Motion, Trans. of the A.M.S. 87 (1958), 187-197. MR 21:3051

Department of Mathematics, Technion-Israel Institute of Technology, Haifa, 32000 ISRAEL

E-mail address: pinsky@techunix.technion.ac.il 